\title{
Rapid production of SaCas9 in plant-based cell-free lysate for activity testing
}

\author{
Andreas Schiermeyer ${ }^{1}$, Pedro Cerda-Bennasser ${ }^{2}$, Thomas Schmelter ${ }^{2}$, Xin Huang ${ }^{3}$, Paul \\ Christou $^{3}$, and Stefan Schillberg ${ }^{4}$ \\ ${ }^{1}$ Fraunhofer-Institut für Molekularbiologie und Angewandte Oekologie Bereich \\ Molekularbiologie \\ ${ }^{2}$ Affiliation not available \\ ${ }^{3}$ University of Lleida School of Agricultural and Forestry Engineering \\ ${ }^{4}$ Fraunhofer-Institut für Molekularbiologie und Angewandte Oekologie IME
}

October 31, 2021

\begin{abstract}
Cas9 nucleases have become the most versatile tool for genome editing projects in a broad range of organisms. The recombinant production of Cas9 nuclease is desirable for in vitro activity assays or the preparation of ribonucleoproteins (RNPs) for DNAfree genome editing approaches. For the rapid production of Cas9, we explored the use of a recently established cell-free lysate from tobacco (Nicotiana tabacum L.) BY-2 cells. Using this system, the 130-kDa Cas9 nuclease from Staphylococcus aureus (SaCas9) was produced and subsequently purified via affinity chromatography. The purified apoenzyme was supplemented with ten different sgRNAs, and the nuclease activity was confirmed by the linearization of plasmid DNA containing cloned DNA target sequences.
\end{abstract}

\section{Introduction}

The precise modification of chromosomal DNA sequences by genome editing offers a wide range of applications in basic research and applied biotechnology. The CRISPR-Cas system has gained widespread acceptance due to its easy implementation. In contrast to other programmable nucleases (e.g., ZFN or TALEN), the CRISPR-Cas system does not require complex protein engineering, as the sequence specificity is brought about by a guide RNA that is easy to design and modify. To deliver the components into the target tissue or cell, various approaches have been used: delivery of DNA constructs coding for Cas9 and sgRNA, delivery of the corresponding transcripts, or delivery of preassembled RNPs [1]. The delivery of DNA constructs usually leads to the integration of the expression cassette into the host genome and therefore a constant presence of the Cas9 nuclease and the sgRNA molecules. However, this may result in undesired effects, such as gene inactivation or rearrangements at the integration site. The delivery of RNA transcripts provides only transient expression of Cas9, as RNA molecules usually have a short half-life. In contrast, preassembled RNPs consisting of single guide RNAs (sgRNA)s and recombinant Cas9 nucleases are not integrated into the host genome, are more stable than transcripts, and usually lead to higher editing efficiencies [2]. To date, the production of recombinant Cas9 nuclease relies on E. coli strains as production hosts followed by purification of the enzyme [3].

The most commonly used Cas9 nucleases are derived fromStreptococcus pyogenes (SpCas9) and Staphylococcusaureus (SaCas9). Although the two nucleases catalyze the same reaction, their properties are different. Both enzymes introduce a DNA double strand break through the action of the two nuclease domains RuvC and HNH. However, the SaCas9 enzyme, consisting of 1053 amino acids, is significantly smaller than the 
SpCas9 enzyme, with 1368 amino acids [4]. Both nucleases cleave in close proximity to the protospacer adjacent motif (PAM), which is defined as 5'-NGG for SpCas9 [5] and 5' NNGRRT for SaCas9 [6], although the molecular mechanisms of cleavage vary between the two enzymes $[7,8,9]$.

Here, we demonstrate that tobacco BY-2 cell-free lysate (BYL) is a powerful platform to provide recombinant SaCas9 at sufficient quantity and quality to verify its nuclease activity on specified DNA target sequences. In particular, we used this assay to validate the nuclease activity of rSaCas9 on target sites within the promoter region of the maize gene coding for carotenoid cleavage dioxygenase 8 (CCD8). Thus, the results of this rapid in vitro assay provided valuable data before embarking on in planta experiments.

\section{Materials and methods}

\subsection{Cloning of the SaCas9 expression construct}

The coding sequence for Cas9 nuclease from S. aureus (GenBank HE980450) has been supplemented with nuclear localization sequences (NLS) derived from SV40 large T antigen and from nucleoplasmin according to Li et al. [10] for subsequent use in planta and a Strep-tag II sequence [11] to facilitate purification of the recombinant protein. The coding sequence was codon-optimized for expression in Zea mays L. using the tool offered by Integrated DNA Technologies Inc. (IDT; https://eu.idtdna.com/CodonOpt). Synthetic DNA fragments (gBlocks) were purchased from IDT, and the coding sequence was merged by Gibson assembly using NEBuilder HiFi DNA Assembly Master Mix (NEB, Frankfurt/Main, Germany). The construct was subcloned into a modified pIVEX vector behind the T7 promoter [12]. The integrity of the construct was verified by Sanger sequencing. The nucleotide and amino acid sequences of rSaCas9 are shown in Figure S1 and S2, respectively.

\subsection{Cloning the target sequence}

The upstream region of the carotenoid cleavage dioxygenase 8 (CCD8) gene [13] from Z. mays L. cv M37W was amplified by PCR from genomic DNA prepared with the EchoLUTION Plant DNA Kit (BioEcho Life Sciences GmbH, Cologne, Germany) using the primer pair Zm_CCD8_for 5'-CAGGCAGCAAACGCTCACCACCAGGC and Zm_CCD8_rev 5'CGACGAAGCCATAGTGGGAGACATGGCG. The amplicon was cloned into pJET1.2 (CloneJET PCR Cloning Kit, Thermo Fisher Scientific) and verified by Sanger sequencing. For subsequent assays, high-quality plasmid DNA was prepared from E. coli harboring pJET_CCD8 using the NucleoBond Xtra Midi Plus kit (Macherey \& Nagel, Düren, Germany).

\subsection{Cloning of the sgRNA expression construct}

Target sites for SaCas9 in the upstream region of the maize CCD8 gene were identified using the Cas-designer tool [14]. Ten target sites were selected (Figure S3), with six sequences derived from the plus strand (T2-T7) and four derived from the minus strand (T1 and T8-T10). Synthetic DNA (gBlocks) comprising the T7 promoter, single guide RNAs (sgRNAs) and a 3' Dra I site (Figure S4) were purchased from IDT (Coralville, Iowa, USA) and subcloned into a modified pIVEX vector [12].

\subsection{In vitro transcription for sgRNA synthesis}

pIVEX_sgRNA vectors were digested with Dra I (NEB) to facilitate run-off sgRNA synthesis. After digestion, the enzyme was inactivated by incubation at $65{ }^{\circ} \mathrm{C}$ for 20 minutes, and the DNA was purified using the MSB Spin PCRapace kit (Invitek Molecular GmbH, Berlin) according to the manufacturer's instructions. In vitro transcription was performed with $1 \mu \mathrm{g}$ of purified DNA template using the HiScribe T7 High-Yield RNA Synthesis Kit (NEB). Reactions were incubated for four hours at $37^{\circ} \mathrm{C}$, and the RNA samples were stored at $-20{ }^{\circ} \mathrm{C}$ until use.

\subsection{Production, purification and analysis of recombinant SaCas9 (rSaCas9)}

The coupled in vitro transcription/translation reaction for the production of rSaCas9 was performed essentially as described [12]. Briefly, $150 \mu$ l of tobacco BY-2 cell-free lysate (BYL, provided by M. Buntru, 
Fraunhofer IME) was placed in individual wells in a 96-well plate. A total volume of $8 \mathrm{ml}$ of BYL was used. Each well was supplemented with $6 \mu \mathrm{g}$ of template DNA (pIVEX_SaCas9). A vector coding for enhanced yellow fluorescent protein (eYFP) was used as a positive control. Empty wells were filled with distilled water to prevent evaporation by increasing the humidity, and the plate was sealed with a lid. The plate was incubated on an orbital shaker (Lab-Therm LT-X, Adolf Kühner AG, Birsfelden, Switzerland) at 500 rpm, $25{ }^{\circ} \mathrm{C}$ and a humidity of $72 \%$ for 48 hours (a sample was taken at $24 \mathrm{~h}$ to monitor protein formation).

After completion of the in vitro transcription/translation reaction, the cell-free reaction mixtures containing rSaCas9 were removed from the wells, combined, and supplemented with 10x washing buffer (Strep-Tactin XT kit, IBA Lifesciences GmbH, Göttingen, Germany) at a ratio of $9+1$. Insoluble material was removed by centrifugation at $16,100 \times g$ for 20 minutes at $4{ }^{\circ} \mathrm{C}$. The supernatant was loaded onto a gravity flow column containing a $1 \mathrm{ml}$ bed volume of Strep-Tactin XT superflow high capacity matrix (IBA) equilibrated with washing buffer. After loading, the matrix was washed with 5 volumes of washing buffer, and protein was eluted subsequently using 3 volumes of elution BXT buffer (IBA). Elution fractions of $0.5 \mathrm{ml}$ (EF\#1 to $\mathrm{EF} \# 6$ ) were collected and stored at $-20{ }^{\circ} \mathrm{C}$.

Protein samples were analyzed by polyacrylamide gel electrophoresis using precast gradient gels (NuPAGE, Bis-Tris, 4-12\%, Thermo Fisher, Waltham, Massachusetts, USA).

\subsection{Activity assay of rSaCas9}

The vector containing the CCD8 promoter target sequence $(0.5 \mu \mathrm{g}$ pJET_CCD8) was mixed with rSaCas9 (150 ng from EF\#2), one of the ten (T1-T10) in vitro transcribed sgRNAs (400-1300 ng) in reaction buffer (NEBuffer1, $10 \mathrm{mM}$ Bis-Tris-Propane- $\mathrm{HCl}, 10 \mathrm{mM} \mathrm{MgCl} 2,1 \mathrm{mM}$ DTT, $\mathrm{pH}$ 7). The reaction mixture (final volume $30 \mu \mathrm{l}$ ) was incubated at $37{ }^{\circ} \mathrm{C}$ for one hour, and aliquots were analyzed by agarose electrophoresis $(0.8 \%(\mathrm{w} / \mathrm{v})$ agarose in TBE, $120 \mathrm{~V}, 30 \mathrm{~min})$.

\section{Results}

To establish the in vitro rSaCas9 activity assay, the enzyme and the sgRNAs specifying the target sites must be produced, and the target DNA substrate must be prepared. The individual steps of the workflow are depicted in Figure 1.

\subsection{Production and purification of $\mathrm{rSaCas} 9$}

To provide sufficient amounts of recombinant SaCas9 for subsequent activity assays, the target protein was produced by coupled in vitro transcription/translation in the cell-free BYL lysate. The ready-to-use BYL reaction contains all required compounds for in vitro transcription and translation, including the T7 polymerase initiating transcription [12]. Only the template vector carrying the expression cassette encoding rSaCas9 has to be added, allowing easy and rapid protein biosynthesis. The recombinant SaCas9 enzyme with additional NLS and Strep-tag II (Figure S2) has a calculated molecular mass of $129.2 \mathrm{kDa}$. SDSPAGE analysis of crude BYL producing either rSaCas9 or eYFP as a positive control demonstrated the successful production of both recombinant proteins with apparent molecular masses of $130 \mathrm{kDa}$ and 32 $\mathrm{kDa}$, respectively (Figure 2). Both proteins are clearly visible as prominent bands within the background of tobacco host proteins. The whole process lasted $48 \mathrm{~h}$, but by $24 \mathrm{~h}$, the production of both recombinant proteins could be observed.

The rSaCas9 nuclease was purified using the C-terminally attached Strep-tag II for affinity chromatography on a Strep-Tactin matrix. This single-step purification protocol yielded a highly pure rSaCas9 apoenzyme, as no major contaminating bands were visible in the elution fractions (EF\#1-EF\#6) on the acrylamide gel. The highest enzyme concentrations were observed in elution fractions EF\#2 and EF\#3 (Figure 3). The purified rSaCas9 protein has an apparent molecular mass of $130 \mathrm{kDa}$, demonstrating the integrity of the enzyme and the absence of proteolytic degradation. The presence of an rSaCas9 moiety in the pellet fraction suggested that a proportion of rSaCas9 is insoluble and thus precipitates. The flow-through and wash fractions also contained rSaCas9, indicating that a portion of SaCas9 was either not bound or only weakly bound to the matrix. Purified protein from EF\#2 was used for subsequent activity assays on target sequences. 


\subsection{Activity assay of rSaCas9}

The activity assay of rSaCas9 is based on sequence-specific cleavage (linearization) of plasmid DNA. Therefore, cell-free produced rSaCas9 was tested on ten different target sites within an ${ }^{\sim} 1 \mathrm{~kb}$ promoter sequence of the CCD8 gene from Z. mays L. Suitable target sites within that sequence were identified using the Cas designer tool (http://www.rgenome.net/cas-designer/). Figure S3 shows the target sites, including the PAM sequence 5'-NNGRRT-3'. Six target sites were derived from the plus strand, and four sites were derived from the minus strand. The corresponding constructs for the in vitrotranscription of the analogous sgRNAs are shown in Figure S4. For the activity assay, the rSaCas9 enzyme was incubated with the individual sgRNAs and the plasmid containing the target sequence. Agarose gel electrophoresis confirmed that the plasmid DNA had been cleaved (linearized) in the presence of each of the ten sgRNAs and rSaCas9, represented by the expected $4 \mathrm{~kb}$ band (Figure 3). In contrast, the control samples without rSaCas9 or without the addition of sgRNA showed no signs of cleavage, as indicated by the higher mobility on the agarose gel due to the more compact conformation of the noncleaved plasmid DNA.

\section{Discussion}

Our experimental data demonstrate the rapid and efficient production of rSaCas9 in the cell-free BYL transcription/translation system (Figure 1) within a time frame of one to two days. Although the molecular mass of rSaCas9 is approximately four times higher than that of the well-established positive control eYFP, its production is clearly visible within crude BYL after gel electrophoresis and protein staining. Enrichment of rSaCas9 and removal of tobacco host proteins was achieved with a single affinity purification step using a C-terminally fused Strep-tag II (Figure 3). The entire procedure of enzyme production and purification is rather simple and only requires a moderate amount of hands-on time. Importantly, the preparation of cell-free lysates and protein biosynthesis can be decoupled. After lysate preparation, ready-to-use reaction samples can be stored at $-80{ }^{\circ} \mathrm{C}$ for up to one year. If necessary, template DNA (plasmid or PCR fragment) carrying the expression cassette encoding the target protein can be added to start protein synthesis. Thus, the cell-free platform provides a flexible, on-demand tool to provide sufficient amounts of Cas9 nuclease for various assays without elaborate and cost-intensive infrastructure for cell-based protein production systems.

With an estimated yield of $0.1 \mathrm{mg}$ of purified rSaCas9, the enzyme amount was sufficient to conduct several hundred in vitro cleavage assays under the conditions used here. However, there is potential to optimize the process and improve enzyme yields further. A fraction of rSaCas9 is insoluble and appears in the pellet fraction during the purification process (Figure 3). To increase the solubility of $\mathrm{rSaCas} 9$, the process parameters of the IVT reaction, such as the incubation temperature, could be adjusted. The affinity purification process resulted in highly pure rSaCas9 (Figure 3), but the enzyme was not captured quantitatively on the matrix, as an $\mathrm{rSaCas} 9$ band is visible on the gel from the flow-through and wash fractions. To improve the enzymematrix interaction, the Strep-tag II might be moved to the other protein terminus, or a Twin-Strep-tag might be used instead [15].

The programmable nuclease activity of purified rSaCas9 was demonstrated by the addition of various sgRNAs that directed the cleavage of ten different target sites within the maize CCD8 promoter sequence (Figure 4). Therefore, we confirmed that this cell-free codon-optimized version of rSaCas9 with additional elements for nuclear targeting is active on selected target sites and can be used for stable transformation experiments in maize to induce mutations in the CCD8 promoter sequence.

The verification of enzyme activity on selected target sites was the primary aim of this study. However, given the simplicity, robustness and speed of the cell-free BYL system, we envisage its use for a variety of other applications in genome engineering projects. These include engineering Cas9 nucleases for higher versatility, e.g., by engineering versions that are less dependent on a PAM sequence [16] [17] for their activity. As in vitro transcription/translation platforms are easily adaptable to high-throughput formats [18, 19], the parallel testing of multiple Cas9 enzymes with altered properties could be achieved. In addition to testing mutant Cas9 variants, the system might also be useful to assess the properties of Cas9 fusion proteins for different purposes, e.g., base editing [20], epigenetic modification [21], or transcriptional activation/repression 
[22]. Once a robust Cas9 nuclease has been identified for a particular purpose, the BYL system can be used to produce RNPs for subsequent DNA-free delivery to the appropriate target cell/tissue.

\section{Acknowledgments}

We are grateful to Dr. Matthias Buntru (Fraunhofer IME) for providing BY-2 cell lysate and valuable advice on in vitrotranscription/translation. Financial support for this research has been provided by the German Federal Ministry of Education and Research (BMBF, grant number 031B0813) and by the Spanish Ministry of Economy and Competitiveness (MINECO), Spain (RTI2018-097613-B-I00; PGC2018-097655-BI00); PROSTRIG, ERA-NET Cofund SusCrop (Grant No 771134).

\section{Conflict of interest}

Stefan Schillberg is a member of the Scientific Advisory Board of LenioBio GmbH distributing the tobacco BY-2 cell-free lysate (BYL) developed by Fraunhofer IME and Corteva.

\section{Data Availability Statement}

Data available on request from the authors.

\section{Author Contributions}

AS, PC, and SS conceived the study.

AS, PCB, XH, and TS conducted experiments and collected data.

AS wrote the manuscript

All authors interpreted the data and proofread and approved the manuscript.

\section{References}

[1] Kouranova, E., Forbes, K., Zhao, G., Warren, J., Bartels, A., Wu, Y., Cui, X. (2016). CRISPRs for optimal targeting: Delivery of CRISPR components as DNA, RNA, and protein into cultured cells and single-cell embryos. Hum. Gene Ther. , 27, 464-475. DOI: 10.1089/hum.2016.009.

[2] DeWitt, M. A., Corn, J. E., Carroll, D. (2014). Genome editing via delivery of Cas9 ribonucleoprotein. Methods , 121-122, 9-15.DOI: 10.1016/j.ymeth.2017.04.003.

[3] Anders, C., Jinek, M. (2014). In vitro enzymology of Cas9.Meth . Enzymol , 546, 1-20. DOI: 10.1016/B9780-12-801185-0.00001-5.

[4] Nishimasu, H., Cong, L., Yan, W. X., Ran, F. A. Zetsche, B., Li, Y., .. Nureki, O et al. (2015). Crystal structure of Staphylococcus aureus Cas9. Cell , 162, 1113-1126. DOI: 10.1016/j.cell.2015.08.007.

[5] Mojica, F. J. M., Díez-Villaseñor, C., García-Martínez, J., Almendros, C. (2009). Short motif sequences determine the targets of the prokaryotic CRISPR defence system. Microbiology , 155, 733-740. DOI: 10.1099/mic.0.023960-0.

[6] Friedland, A. E., Baral, R., Singhal, P., Loveluck, K., Shen, S., Sanchez, M., .. Bumcrot, D. (2015). Characterization of Staphylococcus aureus Cas9: A smaller Cas9 for all-in-one adeno-associated virus delivery and paired nickase applications. Genome Biol ., 16, 257.DOI: 10.1186/s13059-015-0817-8.

[7] Zuo, Z., Liu, J., (2016). Cas9-catalyzed DNA cleavage generates staggered ends: Evidence from molecular dynamics simulations.Sci . Rep ., 5, 37584. DOI: 10.1038/srep37584.

[8] Stephenson, A. A., Raper, A. T., Suo, Z. (2018). Bidirectional degradation of DNA cleavage products catalyzed by CRISPR/Cas9. J. Am. Chem. Soc ., 140, 3743-3750. DOI: 10.1021/jacs.7b13050.

[9] Yourik, P., Fuchs, R. T., Mabuchi, M., Curcuru, J. L. Robb, G. (2019). Staphylococcus aureus Cas9 is a multiple-turnover enzyme. $R N A, 25,35-44$. DOI: 10.1261/rna.067355.118. 
[10] Li, J.-F., Norville, J. E., Aach, J., McCormack, M. Zhang, D., Bush, J., .. Sheen, J. (2013). Multiplex and homologous recombination-mediated genome editing in Arabidopsis and Nicotiana benthamiana using guide RNA and Cas9. Nat. Biotechnol. , 31, 688-691. DOI: 10.1038/nbt.2654.

[11] Witte, C. P., Noel, L., Gielbert, J., Parker, J. Romeis, T. (2004). Rapid one-step protein purification from plant material using the eight-amino acid StrepII epitope. Plant Mol Biol, 55 , 135-147.

[12] Buntru, M., Vogel, S., Stoff, K., Spiegel, H., Schillberg, S. (2015). A versatile coupled cell-free transcription-translation system based on tobacco BY-2 cell lysates. Biotechnol. Bioeng. , 112, 867-878. DOI: $10.1002 /$ bit.25502.

[13] Guan, J. C., Koch, K. E., Suzuki, M., Wu, S. Latshaw, S., Petruff, T., .. McCarty, D. R. (2012). Diverse roles of strigolactone signaling in maize architecture and the uncoupling of a branching-specific subnetwork. Plant Physiol. , 160, 1303-1317. DOI: 10.1104/pp.112.204503.

[14] Park, J., Bae, S., Kim, J.-S. (2015). Cas-Designer: a web-based tool for choice of CRISPR-Cas9 target sites. Bioinformatics, 31,4014-4016. DOI: 10.1093/bioinformatics/btv537.

[15] Yeliseev, A., Zoubak, L., Schmidt, T. G. M. (2016). Application of Strep-Tactin XT for affinity purification of Twin-Strep-tagged CB2, a G protein-coupled cannabinoid receptor. Protein Expres. Purif ., 131, 109-118. DOI: 10.1016/j.pep.2016.11.006.

[16] Walton, R. T., Christie, K. A., Whittaker, M. N., Kleinstiver, B. P. (2020). Unconstrained genome targeting with near-PAMless engineered CRISPR-Cas9 variants. Science , 368, 290-296. DOI: $10.1126 /$ science.aba8853.

[17] Hu, J. H., Miller, S. M., Geurts, M. H., Tang, W., Chen, L., Sun, N., .. Liu, D. R. (2018). Evolved Cas9 variants with broad PAM compatibility and high DNA specificity. Nature, 556, 57, DOI: 10.1038/nature26155.

[18] Chiba, C. H., Knirsch, M. C., Azzoni, A. R., Moreira, A. R., Stephano, M. A. (2021). Cell-free protein synthesis: advances on production process for biopharmaceuticals and immunobiological products.Biotechniques. DOI: $10.2144 /$ btn-2020-0155.

[19] Chandrasekaran, A., Singh, A. K. (2014). One-pot, microscale cell-free enzyme expression and screening, in: Alexandrov, K., Johnston, W. A. (Ed.). Cell-free protein synthesis: Methods and protocols / edited by Kirill Alexandrov and Wayne A. Johnston, Institute for Molecular Bioscience, The University of Queensland, St. Lucia, QLD, Australia, Humana Press, New York, pp. 55-69.

[20] Shimatani, Z., Kashojiya, S., Takayama, M., Terada, R., Arazoe, T., Ishii, H., .. Kondo, A. (2017). Targeted base editing in rice and tomato using a CRISPR-Cas9 cytidine deaminase fusion. Nat. Biotechnol. , 35, 441-443, DOI: $10.1038 /$ nbt.3833.

[21] Nakamura, M., Gao, Y., Dominguez, A. A., Qi, L. S. (2021). CRISPR technologies for precise epigenome editing. Nat. Cell Biol., 23, 11-22, DOI: 10.1038/s41556-020-00620-7.

[22] Shakirova, K. M., Ovchinnikova, V. Y., Dashinimaev, E. B. (2020). Cell reprogramming with CRISPR/Cas9 based transcriptional regulation systems. Front. Bioeng. Biotechnol., 8, 882, DOI: 10.3389/fbioe.2020.00882.

\section{Figure legends}

\section{Figure 1. Process overview}

The whole process for rSaCas9 production and assessment comprises four steps. The first step involves the cloning of constructs for the production of rSaCas9 and sgRNAs, as well as the DNA target sequence. The actual formation of $\mathrm{rSaCas} 9$, different sgRNAs, and the preparation of plasmid DNA containing the target sequence are depicted in the second column. Step three consists of the formation of the RNP complex and 
the enzyme activity test on target DNA. The final step is the analysis of the activity assay. Created with BioRender.com.

\section{Figure 2. Production of rSaCas9 in BYL}

Coupled in vitro transcription/translation was performed in cell-free BYL to produce rSaCas9. The transcription and translation of eYFP served as a positive control. A reaction without DNA template (NTC: no template control) was used as a negative control. After reaction times of $24 \mathrm{~h}$ and $48 \mathrm{~h}, 0.5 \mu \mathrm{l}$ aliquots were separated by SDS-PAGE. Protein bands were stained by Coomassie Brilliant Blue. The filled arrowhead points to rSaCas9, and the open arrowhead indicates eYFP.

\section{Figure 3. Purification of rSaCas9 by affinity chromatography}

The rSaCas9 apoenzyme was purified via its C-terminal Strep-tag II by affinity chromatography on a StrepTactin matrix. Aliquots of $0.5 \mu \mathrm{l}$ (IVT pool, pellet, load, flow through, wash) or $6.5 \mu \mathrm{l}$ (EF\#1 - EF\#6) of the different fractions were separated by SDS-PAGE, and the proteins were visualized by staining with Coomassie Brilliant Blue. Purified rSaCas9 migrates with an apparent molecular mass of $130 \mathrm{kDa}$.

Figure 4. Linearization of plasmid DNA by target site cleavage with rSaCas9

Plasmid DNA containing cloned target sequences (pJET_CCD8) was incubated with purified rSaCas9 and one of ten different sgRNAs (T1-T10) for site-directed DNA cleavage. Fifteen-microliter aliquots of the cleavage reactions were loaded on an agarose gel, and nucleic acids were visualized by ethidium bromide staining. Samples without enzyme (w/o rSaCas9) or without guide RNA (w/o sgRNA) served as controls. The closed arrowhead indicates the slower-migrating linearized plasmid, and the open arrowhead indicates the undigested plasmid DNA.

Figure 1

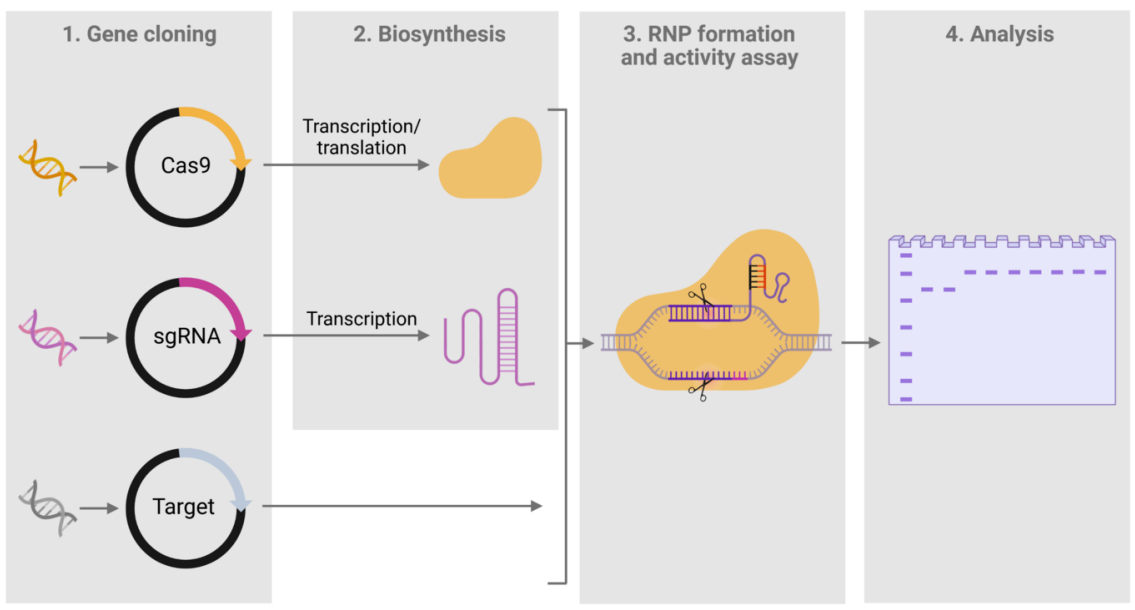

Figure 2 


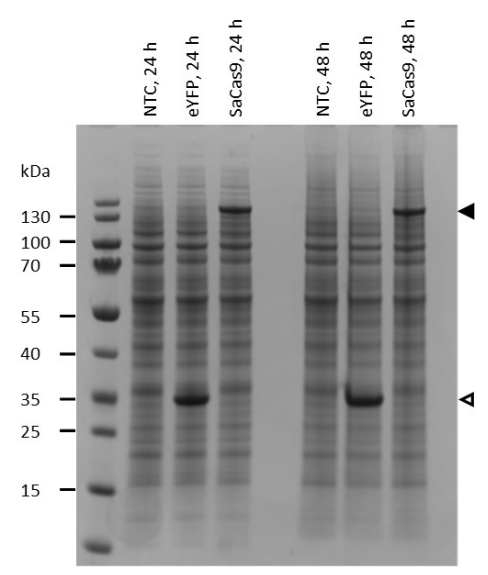

Figure 3 


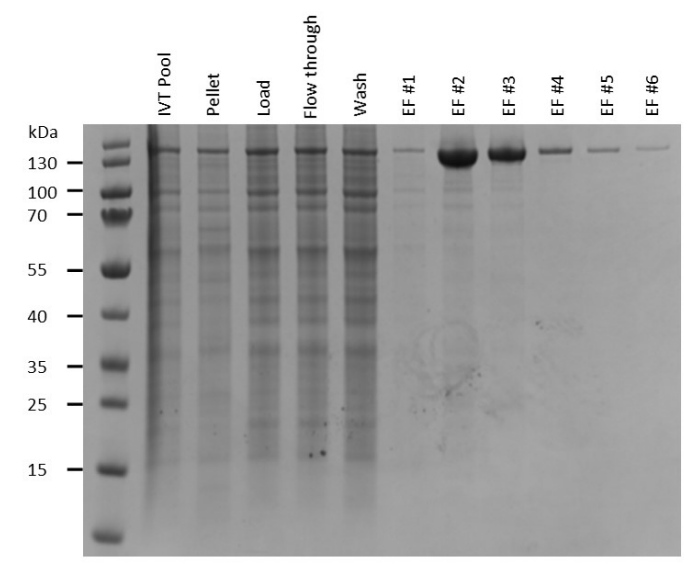

Figure 4 


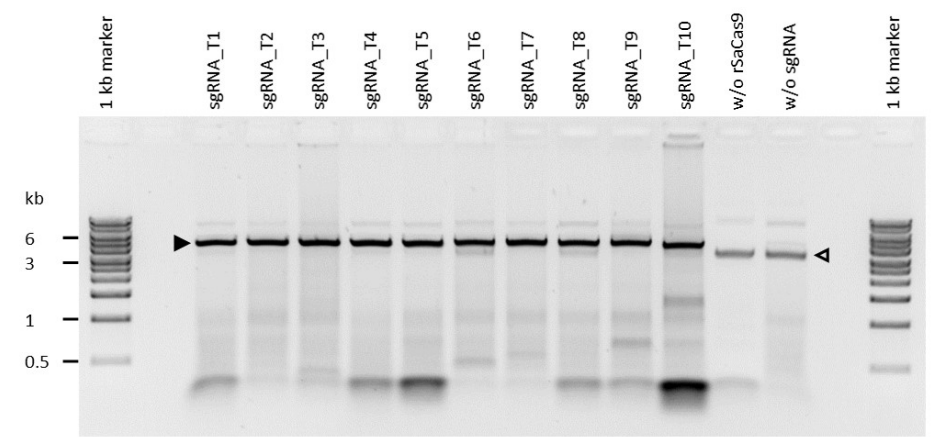

